\title{
Hyperspectral image denoising based on low-rank coefficients and orthonormal dictionary
}

\author{
Fanlong Zhang ${ }^{a, *}$, Guowei Yang $^{a}$ and Jing-Hao Xue ${ }^{b}$ \\ ${ }^{a}$ School of Information Engineering, Nanjing Audit University, Nanjing 211815, China \\ ${ }^{b}$ Department of Statistical Science, University College London, London WCIE 6BT, UK
}

\section{ARTICLE INFO}

\section{Keywords:}

hyperspectral image

denoising

low rank

orthonormal dictionary

alternating minimization

\begin{abstract}
A B S T R ACT
Hyperspectral images (HSIs) are unavoidably contaminated by noise during data acquisition and transmission. A variety of noise reduction approaches have been developed for HSIs, in which the low-rank based methods have emerged as a powerful tool. However, most of low-rank based HSI denoising methods are designed to explore the low-rankness in the pixel space under a fixed dictionary, without seeking the optimal dictionary and exploiting the low-rankness of representation coefficients. In this work, a novel HSI denoising model based on Low-Rank Coefficients and Orthonormal Dictionary (LRCOD) is proposed. The novelty of this work lies in LRCOD's exploiting the low-rankness of representation coefficients and learning an orthonormal dictionary for HSI denoising. To solve the proposed model, an efficient alternating minimization algorithm is developed, since one sub-problem has a closed-form solution and the other can be efficiently solved by the curvilinear search algorithm. Compared with gradient-type algorithms, our algorithm is easy to implement as there is no need to tune optimization parameters like step sizes. The experimental results over both simulated and real datasets verified the superiority of the proposed method. Specifically, under various levels of noise in different scenes, LRCOD yields the best MPSNR in 30 of 35 cases and runs faster than other methods except one non-iterative algorithm.
\end{abstract}

\section{Introduction}

Hyperspectral image (HSI) captures the reflected or emitted electromagnetic energy from a scene over hundreds of narrow, contiguous spectral bands, from visible to infrared wavelengths. It has a wide range of applications in agriculture, mineral exploration, environmental monitoring, weather prediction, food safety, biomedicine and forensics [1].

HSI is a three-dimensional data cube, in which the first two dimensions represent spatial information and the third dimension represents the spectral information of a scene. In real applications, the observed HSI has been degraded by various sources of noise, which severely influences the image's visual appearance and limits the performance of subsequent HSI analysis tasks, such as classification [2-7], target detection [8-11] and unmixing [12-15]. Therefore, the noise free HSI needs to be estimated; this task is referred to as denoising, also called restoration or noise reduction.

Past two decades witnessed high growth in the number of papers on HSI denoising [16]. If we regard each spectral band as an independent gray-level image, the traditional denoising methods, such as block matching and 3-D filtering (BM3D) [17], can be directly applied to the HSI in a bandby-band manner. However, the correlation among spectral bands is ignored in this case, resulting in degraded performance for HSI denoising. Thus, numerous denoising approaches considering the spatial-spectral structure of HSI have been developed under different frameworks, such as sparse representation based methods [18, 19], and low-rank matrix approximation based methods [16, 20-24].

\footnotetext{
*Corresponding author

@ csfzhang@126.com (F. Zhang)

ORCID(s):
}

Among them, low-rank matrix approximation has emerged as a powerful tool for HSI analysis. It finds and exploits low-dimensional structures in high-dimensional data. Due to the redundancy in the spectral bands, a clean HSI has been widely approximated by a low-rank matrix. The aim of lowrank based denoising methods for HSI is to remove the noise in the polluted image to recover the clean image.

Given an observed HSI $\mathbf{Y} \in R^{n \times m}$, with $n$ being the number of bands, $m=h w$ being the number of pixels, and $(h, w)$ being the height and width of each band, a widely used assumption is that $\mathbf{Y}$ can be represented as a combination of true unknown signal $\mathbf{X}$ and additive noise $\mathbf{N}$ :

$$
\mathbf{Y}=\mathbf{X}+\mathbf{N} \text {. }
$$

Due to high correlation among clean HSI spectral bands [20, 21], one often assumes that the spectral signature vectors (columns of $\mathbf{X}$ ) lie in a low-dimensional subspace. Thus, the clean $\mathbf{X}$ can be decomposed as $\mathbf{X}=\mathbf{E Z}$, where $\mathbf{E} \in R^{n \times s}$ represents the matrix of $s$ basis vectors of the subspace and $\mathbf{Z} \in R^{s \times m}$ denotes the matrix of representation coefficients.

HSI denoising methods based on the low-rank assumption can be divided to two categories. One is based on patches of HSI, and the other is based on the whole HSI. Methods based on patches extract similar non-local patches from $\mathbf{X}$ or representation coefficient matrix $\mathbf{Z}$, and rearrange these patches into a new matrix $\overline{\mathbf{X}}$ [22] or $\overline{\mathbf{Z}}$ [21], which are assumed to being low-rank. However, finding similar patches or tensors is time consuming. In addition, to exploit the nonlocal similarity over space and the global correlation across spectrum, tensor low-rank models have been employed for HSI denoising. For example, a low-rank tucker approximation is enforced on each group (i.e., a tensor) separately [25]; a simultaneously sparse and low-rank structure is considered 
for each tensor of an HSI in [26]. However, both methods only capture intra-tensor information without exploiting inter-tensor correlations. Thus, [27] presents an effective tensor dictionary learning method for HSI restoration by exploiting the global sparsity through shared dictionaries among different tensors.

Due to the redundancy in the spectral bands, low-rank methods based on the whole HSI assume a much lower spectral dimension than the original HSI, i.e. $s \ll \min (m, n)$. Specifically, the term $\mathbf{E}$ is called dictionary, which is extracted from the HSI itself. Normally, the rows of observed HSI $\mathbf{Y}$ are highly correlated and it means that the matrix $\mathbf{Y}$ is a low-rank matrix. Thus, the corresponding coefficient matrix $\mathbf{Z}$ is also low-rank. Thus, many models pursuing lowrank $\mathbf{Z}$ have been established [28, 29]. However, they are mainly used for solving HSI unmixing problems, and extra constraints, including the sum-one constraint and the nonnegative constraint, are imposed on corresponding models. Recently, on account of the powerful feature extraction and nonlinear expression ability brought by deep neural networks, [30] proposes a spatial-spectral gradient network for hybrid noise reduction in HSI, where the spatial gradient and the spectral gradient are jointly incorporated. Furthermore, [31] proposes an alternating directional three-dimensional quasirecurrent neural network for HSI denoising, which can effectively model spatio-spectral dependency.

Differing from them, this paper proposes a novel HSI denoising model based on Low-Rank Coefficients and Orthonormal Dictionary (LRCOD); the main novelties of this work can be summarized as follows.

(i) The low-rankness of the coefficient matrix $\mathbf{Z}$ is explored for denoising. The low-rankness of coefficient matrix is also explored for HSI unmixing [28]. The difference between our work and [28] is that: in unmixing, the extra constraints of coefficient matrix are necessary, which are the sum-one constraint and the non-negative constraint, as each coefficient vector denotes the fractional abundances of the endmembers in unmixing.

(ii) An optimal dictionary $\mathbf{E}$ is learned under an orthonormal constraint, which means that column vectors of $\mathbf{E}$ are all unit vectors and orthogonal to each other. This constraint has at least four merits. a) We generalize the fixed orthonormal dictionary to a learned one, by minimizing the representation error $\|\mathbf{E Z}-\mathbf{Y}\|_{F}^{2}$. b) The unity can avoid dictionary atoms reaching arbitrarily high values as previous unit-norm constraint doing. c) The orthogonality can transfer the low rankness of HSI to the coefficients matrix. d) The orthogonality can benefit solving the model, as detailed in Section 3.

The rest of this paper is organized as follows. Section 2 details our proposed LRCOD model, as well as its relationship with other models. In Section 3 we solve LRCOD by presenting an alternating minimization algorithm. Section 4 compares LRCOD with the state of the art on simulated and real datasets. Section 5 draws conclusions for this work.

\section{Model and Motivation}

First, we establish some notation. The numbers of bands and pixels in each band are denoted by $n$ and $m$, respectively, where $m=h \times w$ with $h$ and $w$ being the height and width, respectively, of each band. Matrices are denoted by bold and capital letters, column vectors by bold letters, scalars by italic letters. The identity matrix of size $p \times p$ is denoted by $\mathbf{I}_{p \times p}$, simplified as $\mathbf{I}$ without causing confusion. The $\mathbf{A}^{T}$ stands for the transpose matrix of $\mathbf{A}$. Given a matrix $\mathbf{X}$, its $(i, j)$-th entry will be noted as $x_{i j}$, and operators used include Frobenius norm $\|\mathbf{X}\|_{F}$ obtained by $\sqrt{\sum_{i, j} x_{i j}^{2}}$ and nuclear norm $\|\mathbf{X}\|_{*}$ obtained by $\sum_{i} \sigma_{i}(\mathbf{X})$, i.e., the sum of the singular values of $\mathbf{X}$.

\subsection{Problem setting}

An observed HSI $\mathbf{Y} \in R^{n \times m}$ contaminated by noise can be represented as Eq.(1), where $\mathbf{X}$ is the unknown clean signal which needs to be estimated, and $\mathbf{N}$ a matrix representing the noise. Furthermore, one usually assumes that the spectral vectors lie in a low-dimensional subspace, which is a very good approximation for most real HSI [32]. Therefore, we can rewrite Eq.(1) as

$$
\mathbf{Y}=\mathbf{E Z}+\mathbf{N},
$$

where $\mathbf{E} \in R^{n \times s}$ is called the basis matrix or dictionary, and $\mathbf{Z} \in R^{s \times m}$ is the matrix of representation coefficients of $\mathbf{Y}$ with respect to $\mathbf{E}$. Now, the problem becomes how to pursue $\mathbf{E}, \mathbf{Z}$ and $\mathbf{N}$. Next, we propose our solution and its motivation.

\subsection{Model and motivation}

Based on the representation model (2), we propose a new HSI denoising model based on Low-Rank Coefficients and Orthogonal Dictionary (LRCOD):

$$
\min _{\mathbf{Z}, \mathbf{E}} f(\mathbf{E}, \mathbf{Z}):=\frac{1}{2}\|\mathbf{E} \mathbf{Z}-\mathbf{Y}\|_{F}^{2}+\lambda\|\mathbf{Z}\|_{*} \text { s.t. } \mathbf{E}^{T} \mathbf{E}=\mathbf{I},
$$

where the first term represents the data fidelity; the second term is the low-rank regularization imposed on the representation coefficients; the parameter $\lambda$ determines the trade-off between the two terms; and the constraint term is the orthogonal condition imposed on the representation dictionary. We provide two justifications for the proposed model.

(i) Justification of low-rank assumption for coefficient matrix $\mathbf{Z}$.

Firstly, EZ in model (3) has low rank. Specifically, HSI can be approximated by a low-rank matrix from the view of unmixing, due to the band similarity of HIS [33]. It assumes that the spectral response of a pixel is a linear combination of all the endmembers present in the pixel. In the matrix form, the clean matrix can be factorized as

$$
\mathbf{X}=\mathbf{A B},
$$

where $\mathbf{A} \in R^{n \times k}$ is the endmember matrix, the columns of $\mathbf{B} \in R^{k \times m}$ contain the fractional abundances of the endmembers for all the pixels. In general the number of endmembers 
$k \ll \min (m, n)$. Thus, the denoised HSI, i.e. EZ in model (3), is a low-rank matrix.

Secondly, we show that $\mathbf{Z}$ in model (3) has low rank from two aspects. On the one hand, under the constraint $\mathbf{E}^{T} \mathbf{E}=\mathbf{I}$, we have $\mathbf{Z}=\mathbf{E}^{T} \mathbf{X}$; then, since the matrix $\mathbf{X}$ is latent lowrank, the matrix $\mathbf{Z}$ should also be low-rank. In other words, the orthogonal constraint transfers the low rankness of HSI to the coefficients matrix $\mathbf{Z}$. On the other hand, the dictionary $\mathbf{E}$ usually satisfies the full column rank property [34]. Thus, according to Lemma 1 below, we can infer that the corresponding representation matrix $\mathbf{Z}$ is also low-rank.

Lemma 1 [35] Given matrices $\mathbf{E} \in R^{n \times s}$ and $\mathbf{Z} \in R^{s \times m}$, if $\operatorname{rank}(\mathbf{E Z})=k \leq \min (s, m)$ and $\operatorname{rank}(\mathbf{E})=s$, then we have $\operatorname{rank}(\mathbf{Z})=\operatorname{rank}(\mathbf{E Z})=k$.

(ii) Justification for learning dictionary $\mathbf{E}$ under orthonormal constraint.

In many previous studies, dictionaries $[20,36]$ are inferred from the noisy observation itself through the HySime algorithm [36] or singular value decomposition (SVD). Such dictionaries are orthonormal, fixed and implicit at the procedure of learning the representation coefficients, and cannot obtain the minimum representation error. In our model, differing from the fixed orthonormal dictionary, an optimal dictionary is learned to minimize the representation error.

There are many algorithms to learn the dictionary. Different algorithms for learning dictionaries may result in different efficiency. Most of them constrain the base vector $\mathbf{e}_{i}$ of dictionary to a non-convex unit-norm surface (i.e. $\left\|\mathbf{e}_{i}\right\|_{F}=$ 1) or a convex unit-norm ball (i.e. $\left\|\mathbf{e}_{i}\right\|_{F} \leq 1$ ). The basic purpose of adding such constrains is to avoid dictionary atoms reaching arbitrarily high values. In our model, we constrain the base vectors orthonormal, that is, column vectors of $\mathbf{E}$ are all unit vectors and orthogonal to each other. As a result, there are at least the following benefits.

a) The unity avoids dictionary atoms reaching arbitrarily high values. The orthonormal constraint is originated from the orthonormality of fixed dictionary, and in fact is stronger than unit-norm constraint.

b) The orthonormality can transfer the low-rankness of HSI to the coefficients matrix.

c) The orthonormality benefits the solving of the model. Explicitly, under the constraint $\mathbf{E}^{T} \mathbf{E}=\mathbf{I},\|\mathbf{E Z}-\mathbf{Y}\|_{F}^{2}$ is equivalent to $\left\|\mathbf{Z}-\mathbf{E}^{T} \mathbf{Y}\right\|_{F}^{2}$, as detailed in Section 3 .

\subsection{Relationship with existing methods}

There has been a great deal of research activity on HSI processing based on low-rank assumption. Some clear differences between existing methods and ours are as follows.

Firstly, in many methods [20-22], the low-rank assumption is imposed on the patches-induced matrix. They extract the nonlocal self-similarity patches from $\mathbf{X}$ or $\mathbf{Z}$. Then new matrix formed by patches is assumed being low-rank. In our model, there does not exist the concept of patches. Very importantly, finding similar patches is time consuming, since a patch matching algorithm is needed to search the similar patches for each reference patch.

Secondly, this work has close ties to many HSI denoising models mentioned in [16], where the low-rank assumption is imposed on the HSI itself and thus $\mathbf{X}$ is assumed low-rank. In this case, the redundancy in the spectral bands of HSI is considered and measured by rank size. Differently, our work focus on the redundancy in representation coefficient, and assume the coefficient matrix $\mathbf{Z}$ is low-rank. This opens a new perspective for HSI denoising.

Finally, [28] also exploited the low-rankness of coefficient matrix. However, their model focuses on HSI unmixing, and the extra sum-one and non-negative constraints on coefficient matrix are imposed on the model. These constraints ensure coefficient vector denoting the fractional abundances of the endmembers in unmixing. Furthermore, [37] proposed a superpixel based denoising method, called FS ${ }^{2}$ LRL, by employing the the low-rankness of coefficient sub-matrix. There exist significant differences between FS ${ }^{2}$ LRL and our proposed LRCOD. In LRCOD, the low-rankness of $\mathbf{Z}$ is decided by the band similarity and dictionary. Specifically, HSI can be approximated by a low-rank matrix due to the band similarity, and this low-rankness is transformed into coefficient matrix $\mathbf{Z}$ under the properties of orthonormal constraint and full column rankness of dictionary. In FS ${ }^{2} \mathrm{LRL}$, however, the low-rankness of $\mathbf{Z}_{i}$, a sub-matrix extracted from $\mathbf{Z}$, is decided by the pixel similarity. Specifically, the HSI is segmented into superpixels, and sub-matrix $\mathbf{Z}_{i}$ combined by the coefficients corresponding to the same superpixel $i$ is assumed to be low-rank.

\section{Algorithm}

The optimization problem (3) is a joint optimization problem. One approach to solving (3) is by concatenating $\mathbf{E}$ and $\mathbf{Z}$ as a single variable and then directly applying standard iterative algorithms like gradient descent (or its variants); see [38, 39] for an overview. However, as the variables within the problem are naturally parted into two blocks $\mathbf{E}$ and $\mathbf{Z}$, it can be easier to update just one block variable at a time, without updating all variables simultaneously. The alternating minimization is suitable for such a purpose.

Alternating minimization (also known as block-coordinate minimization) sequentially optimizes over one block variable while fixing the other. Specifically, we update variables $\mathbf{E}$ and $\mathbf{Z}$ alternately as follows.

(i) Given $\mathbf{E}=\mathbf{E}^{k}$, update $\mathbf{Z}$ by solving

$$
\min _{\mathbf{Z}} \frac{1}{2}\|\mathbf{E} \mathbf{Z}-\mathbf{Y}\|_{F}^{2}+\lambda\|\mathbf{Z}\|_{*}
$$

which, on the benefit of constraint $\mathbf{E}^{T} \mathbf{E}=\mathbf{I}$, is equivalent to

$$
\min _{\mathbf{Z}} \frac{1}{2}\left\|\mathbf{Z}-\mathbf{E}^{T} \mathbf{Y}\right\|_{F}^{2}+\lambda\|\mathbf{Z}\|_{*}
$$

The problem (6) has a closed-form solution $D_{\lambda}\left(\mathbf{E}^{T} \mathbf{Y}\right)$ [40]. Here $D_{\lambda}(\cdot)$ is called the singular value shrinkage operator, which is defined by

$$
D_{\lambda}(\mathbf{Q})=\mathbf{U} \operatorname{diag}\left(\left\{\max \left(0, \sigma_{j}-\lambda\right)\right\}_{1 \leq j \leq r}\right) \mathbf{V}^{T},
$$


where $\mathbf{Q}=\mathbf{U S V}^{T}, \mathbf{S}=\operatorname{diag}\left(\sigma_{1}, \cdots, \sigma_{r}\right)$.

(ii) Given $\mathbf{Z}=\mathbf{Z}^{k+1}$, update $\mathbf{E}$ by solving

$$
\min _{\mathbf{E}} \frac{1}{2}\|\mathbf{E Z}-\mathbf{Y}\|_{F}^{2} \quad \text { s.t. } \mathbf{E}^{T} \mathbf{E}=\mathbf{I} .
$$

This is an optimization problem with orthogonality constraints. One can easily obtain the gradient function

$$
\nabla F(\mathbf{E})=\mathbf{E Z Z} \mathbf{Z}^{T}-\mathbf{Y} \mathbf{Z}^{T}
$$

By employing the gradient information, we solve problem (8) by the curvilinear search algorithm [41]. Specifically, given a feasible point $\mathbf{E}^{k}$ and the gradient matrix $\mathbf{G}:=$ $\nabla F\left(\mathbf{E}^{k}\right)$, the search direction $\mathbf{D}^{k}(\tau)$ and step size $\tau^{k}$ of the next iteration point are given by Eqs.(10) and (11) [41]:

$$
\begin{aligned}
& \mathbf{D}^{k}(\tau)=\left(\mathbf{I}+\frac{\tau}{2} \mathbf{H}\right)^{-1}\left(\mathbf{I}-\frac{\tau}{2} \mathbf{H}\right) \mathbf{E}^{k}, \\
& \tau^{k}=\frac{\operatorname{tr}\left(\left(\mathbf{S}^{k-1}\right)^{T} \mathbf{S}^{k-1}\right)}{\left|\operatorname{tr}\left(\left(\mathbf{S}^{k-1}\right)^{T} \mathbf{D}^{k-1}\right)\right|},
\end{aligned}
$$

where $\mathbf{H}=\mathbf{G}\left(\mathbf{E}^{k}\right)^{T}-\mathbf{E}^{k} \mathbf{G}^{T}, \mathbf{S}^{k-1}=\mathbf{E}^{k}-\mathbf{E}^{k-1}$, and $\mathbf{D}^{k-1}=$ $\nabla F\left(\mathbf{E}^{k}\right)-\nabla F\left(\mathbf{E}^{k-1}\right)$.

The curvilinear search algorithm for problem (8) is shown in Algorithm 1. The detailed algorithm for model (3) is summarized in Algorithm 2. Given a tolerance $\epsilon$, we use the relative error as the stopping criterion defined by

$$
\frac{\left\|f\left(\mathbf{E}^{k+1}, \mathbf{Z}^{k+1}\right)-f\left(\mathbf{E}^{k}, \mathbf{Z}^{k}\right)\right\|_{F}}{\left\|f\left(\mathbf{E}^{k}, \mathbf{Z}^{k}\right)\right\|_{F}}<\epsilon .
$$
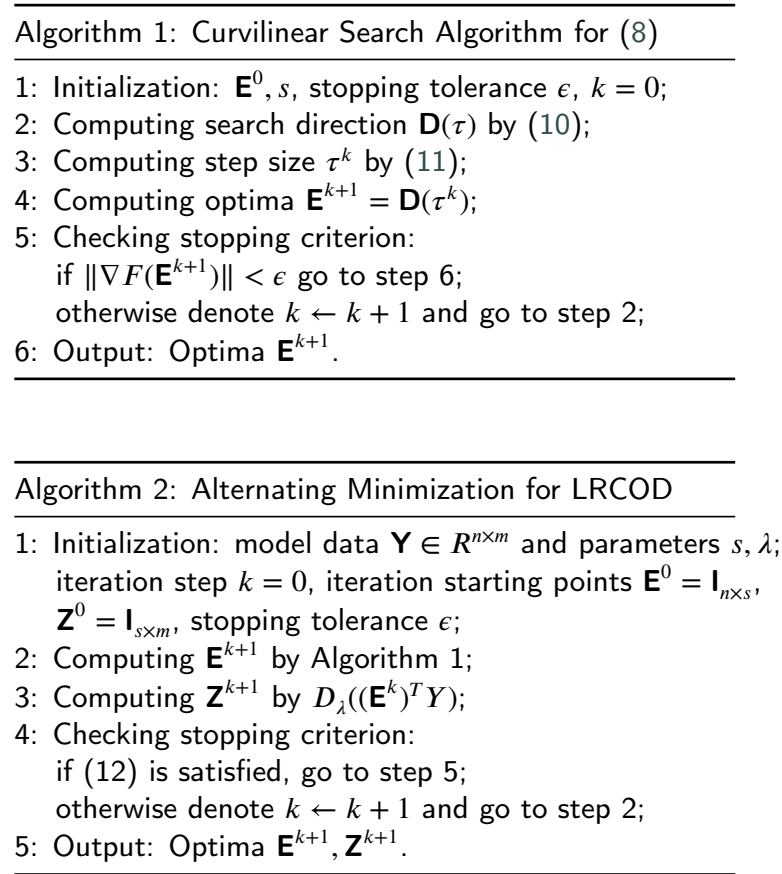

F. Zhang, G. Yang and J.-H. Xue: Preprint submitted to Elsevier
Algorithm merits. Compared with gradient-type algorithms, our algorithm has at least three merits. (i) Each subproblem is easy to solve: one has a closed-form solution, and the other can be solved by the curvilinear search algorithm efficiently. (ii) It is easy to implement as there is no need to tune optimization parameters like step sizes. (iii) It converges very fast in practice. The convergence speed can be observed in Fig.1, where we have plotted the objective function value vs. the iteration number at various levels of noise, as described in Section 4.1. We have found that the algorithm converges quickly for the first 20 iterations, and then becomes stable after about 40 iterations.

Algorithm complexities. Given an observed HSI Y $\in$ $R^{n \times m}$, with $n$ being the number of bands, $m$ being the number of pixels, and $s$ being the number of dictionary atoms, the main computational costs of the LRCOD are due to updating $\mathbf{E}$ and $\mathbf{Z}$. The $\mathbf{Z}$ is updated by SVD of $\mathbf{E}^{T} \mathbf{Y}$, having computational costs of $O\left(m s^{2}\right)$, and the $\mathbf{E}$ is updated by Algorithm 1, having time complexity of $O\left(t n^{3}+t n s^{2}\right)$, where $t$ denotes the iteration number of Algorithm 1. Therefore, our algorithm has computational costs of $O\left(m s^{2}+t n^{3}+t n s^{2}\right)$ for each iteration. As comparison, the computational costs of the LRMR method is $O(m n r p)$ for each iteration, where $p$ is the number of patches and $r$ the upper bound of endmembers. Furthermore, the computational costs of the FastHyDe algorithm [20] are due to learning the subspace $\mathbf{E}$ from $\mathbf{Y}$, having computational costs of $O\left(m n^{2}\right)$, and computing the noisy eigen-images $\mathbf{E}^{T} \mathbf{Y}$, having computational costs of $O\left(m s^{2}\right)$. Thus FastHyDe has computational costs of $O\left(m s^{2}+m n^{2}\right)$.

\section{Experiments}

The quantitative and visual results are evaluated on both synthetic and real HSIs for demonstrating the effectiveness of the proposed algorithm. The proposed algorithm is compared with the state-of-the-art algorithms, including fast hyperspectral denoising algorithm (FastHyDe) [20], subspacebased nonlocal low-rank and sparse factorization algorithm (SNLRSF) [21], non-i.i.d. mixture of Gaussian algorithm based on low-rank matrix factorization (NMoG-LRMF) [42], block-matching and 4D filtering algorithm (BM4D) [43], HSI restoration algorithm on the basis of low-rank matrix recovery (LRMR) [22], and spatio-spectral total variation algorithm (SSTV) [44].

The parameters for these compared methods were manually adjusted according to their default strategies. In addition, to facilitate the numerical calculation and visualization, all the bands of the HSI datasets are normalized into $[0,1]$.

\subsection{Algorithm convergence}

LRCOD starts with some initial guesses of $\mathbf{E}$ and $\mathbf{Z}$ and then alternately updates them. Reasonable initial guesses are application dependent; Fig. 1 shows the convergence after different initialization methods at six different noise levels on the Pavia Centre dataset of size $200 \times 200 \times 103$ by adding uniform noise with intensity of $0.02,0.04,0.06,0.08$, 0.1 and 0.20 , respectively. For visualization, we show the variation of objective function values beginning from the 
Hyperspectral image denoising based on low-rank coefficients and orthonormal dictionary
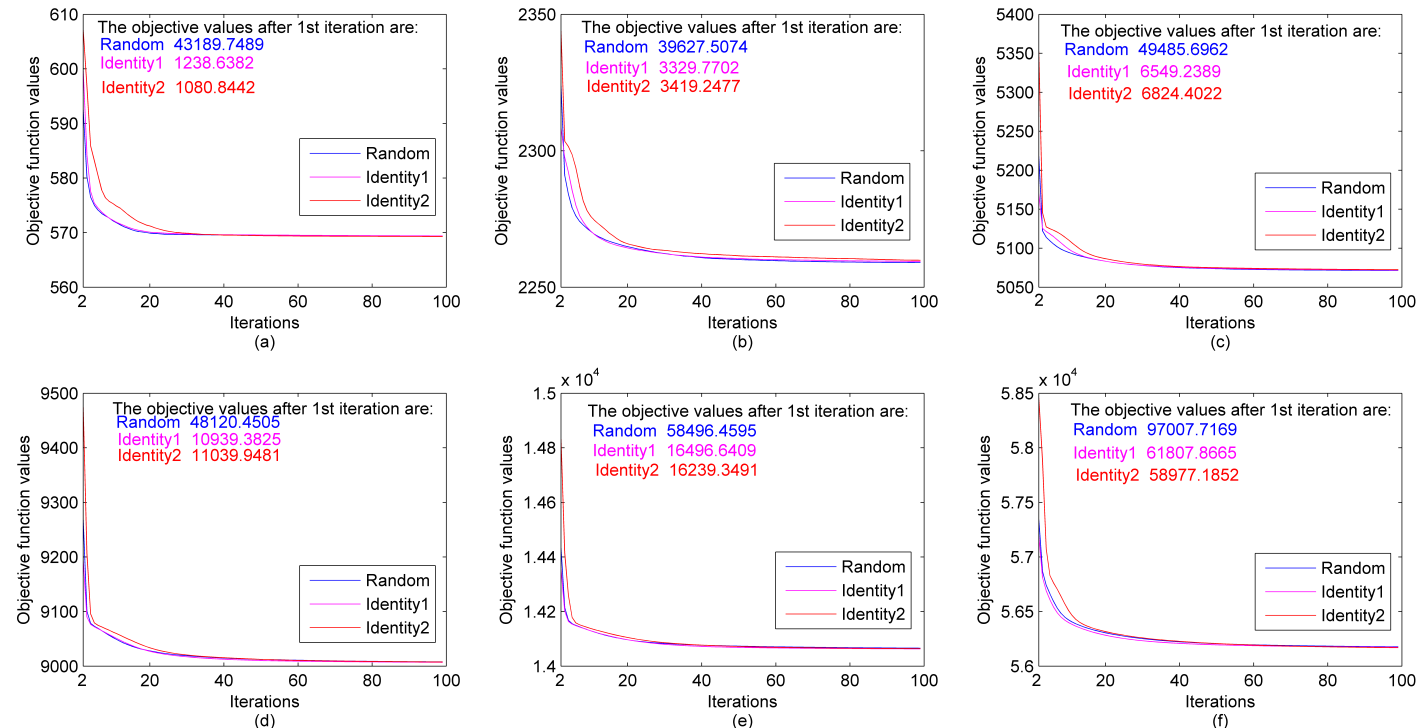

Figure 1: Objective function value versus iteration at noise intensity of (a) 0.02 , (b) 0.04, (c) 0.06 , (d) 0.08 , (e) 0.1 , and (f) 0.2 . Different methods of initialization are used: the first method is to initialize $\mathbf{E}$ and $\mathbf{Z}$ by random matrices ('Random'); the second method is to initialize $\mathbf{E}$ and $\mathbf{Z}$ by corresponding identity matrices ('Identify1'); and the third method is to initialize $\mathbf{E}$ by the identity matrix and $\mathbf{Z}$ by $\mathbf{E}^{T} \mathbf{Y}$ ('Identity2').
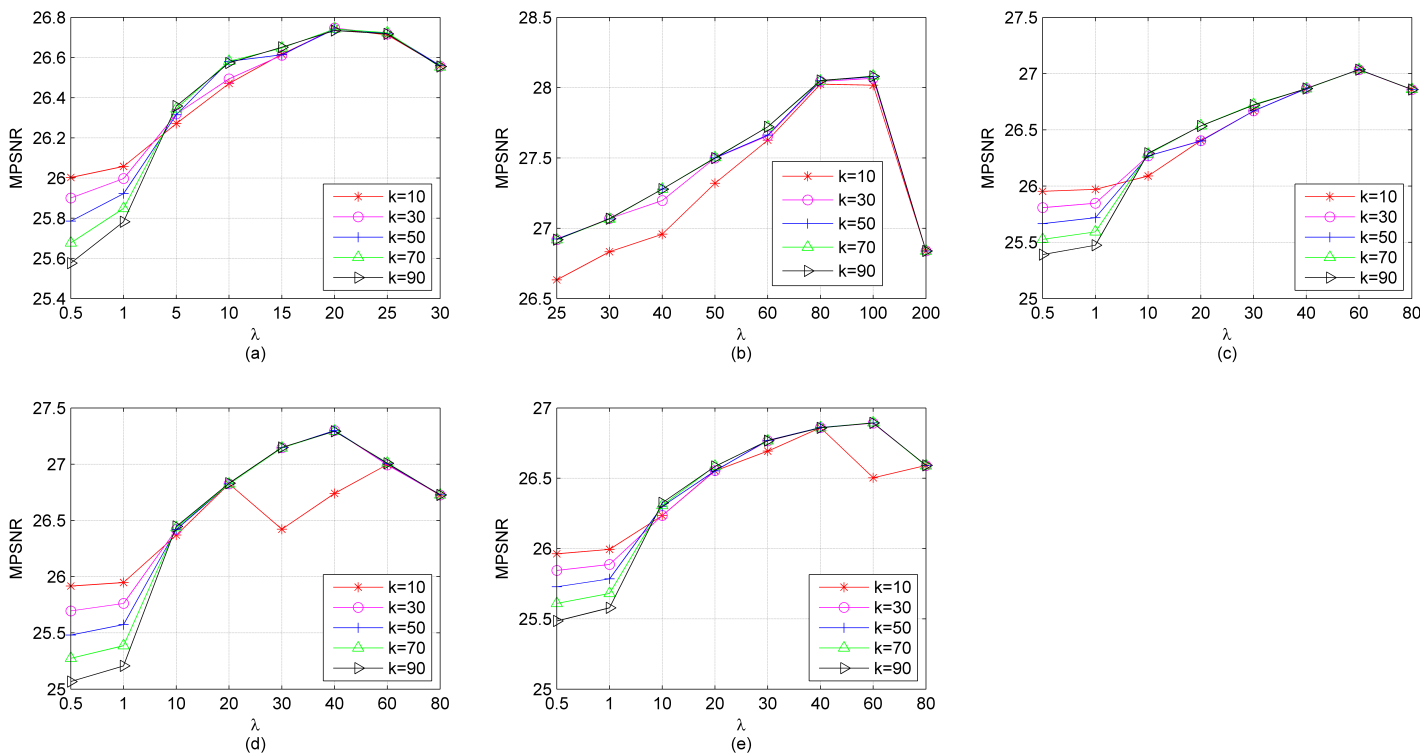

Figure 2: Sensitivity analysis of parameters $\lambda$ and $k$ in terms of MPSNRs on (a) Japser Ridge, (b) Cuprite, (c) Urban, (d) Pavia, and (e) Washington DC.

2nd iteration, and the objective function value after the 1st iteration is noted in figure. We can make the following observations: i) the objective value of the 2 nd iteration is much less than that of the $1 \mathrm{st}$ iteration; ii) no matter of the initialization methods, the objective function becomes stable after about 40 iterations; and iii) generally speaking, the variation of objective function value is less than $10^{-6}$ when the number of iterations is over 30 .

However, the empirical performance of alternating mini- mization is not sufficiently substantiated by convergence guarantees. Although the idea of alternately updating the variables is quite straightforward, the convergence properties for alternating minimization are far more complicated [45].

\subsection{Parameter analysis}

In this section, sensitivity analysis of regularization parameter $\lambda$ and dictionary dimension $k$ are undertaken on the simulated datasets: the Japser Ridge dataset of size $100 \times$ $100 \times 224$ and the Cuprite dataset of size $250 \times 250 \times 224$. 

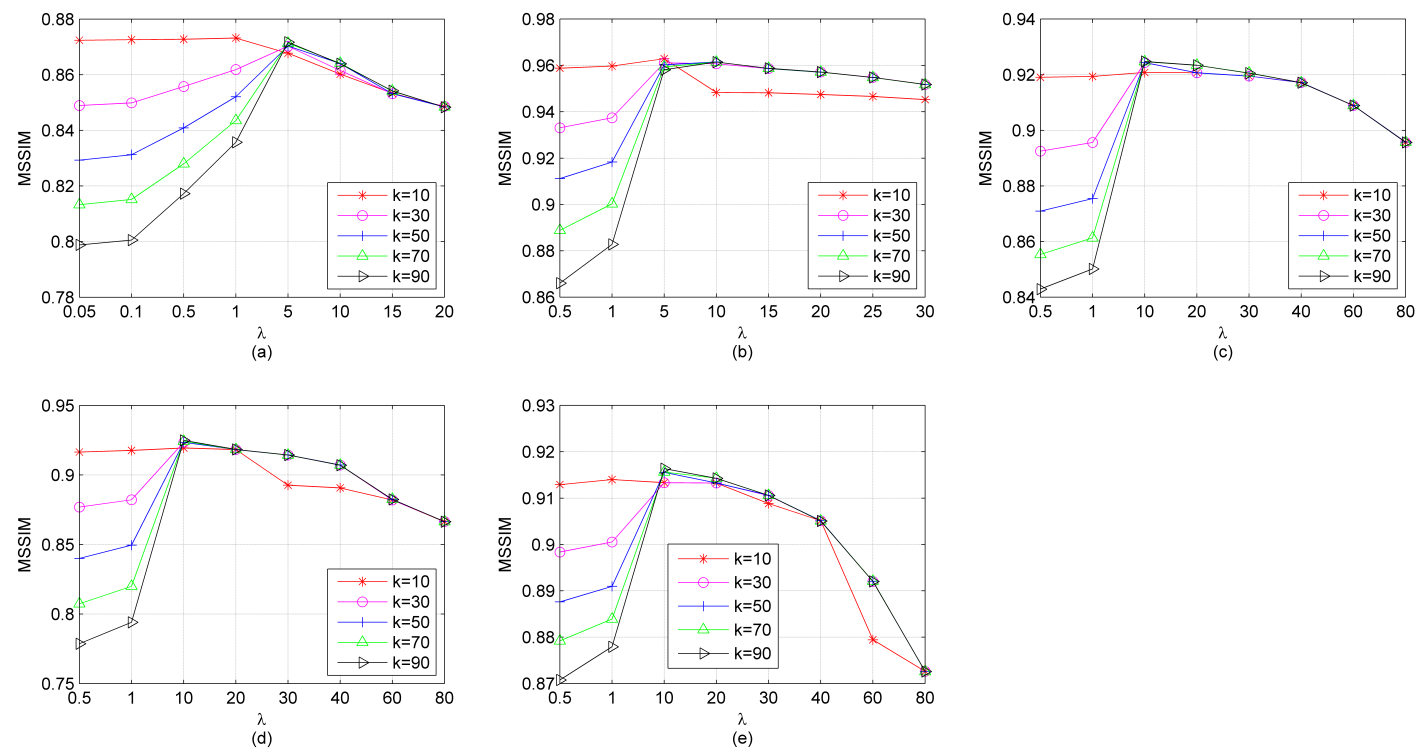

Figure 3: Sensitivity analysis of parameters $\lambda$ and $k$ in terms of MSSIMs on (a) Japser Ridge, (b) Cuprite, (c) Urban, (d) Pavia, and (e) Washington DC.

To simulate the clean HSI, HSIs are firstly denoised via SVD. Then these denoised HSIs are considered as clean data in this simulated experiment. To simulate noisy HSIs, we polluted the clean HSI images by adding uniform noise with intensity of 0.1. The mean peak signal-to-noise ratio (MPSNR) and mean structural similarity index (MSSIM) are employed for quantitative comparison:

$$
\operatorname{MPSNR}=\frac{1}{n} \sum_{i=1}^{n} \operatorname{PSNR}_{i}, \quad \operatorname{MSSIM}=\frac{1}{n} \sum_{i=1}^{n} \operatorname{SSIM}_{i} .
$$

We test $\lambda$ in the interval $[0.001,200]$ and dictionary dimension $k$ in the interval $[10,100]$ on five datasets, which are Japser Ridge, Cuprite, Urban, Pavia Centre, and Washington DC Mall. The denoising results in terms of MPSNR and MSSIM are shown in Fig. 2 and Fig.3.

We can make the following observations. Firstly, under small $\lambda$, the MPSNRs and MSSIMs vary clearly with dimension $k$. Especially, one can obtain the best or competitive performance under large $k$. This observation is very different from that in the unmixing models, where $k$ is explained as the number of endmembers and is usually small. Secondly and interestingly, under large $\lambda$ the performance becomes stable, no matter of $k$. The $\lambda$ determines the trade-off between the data fidelity term and the regularization term, and larger $\lambda$ means less importance of data fidelity, thus $k$ does not have much effect. Finally, the best MPSNR and MSSIN are not corresponding to the same value of parameters, whatever $k$ or $\lambda$. For fair comparison, the MPSNR and MSSIN are obtained under the same parameter setting in following experiments.

\subsection{Experiments on simulated data}

Five HSI datasets are employed in this experiment: Washington DC Mall subscene of size $256 \times 256 \times 191$, Pavia Cen- tre subscene of size $200 \times 200 \times 103$, Urban of size $307 \times$ $307 \times 162$, Cuprite of size $250 \times 250 \times 224$, and Japser Ridge of size $100 \times 100 \times 224$. Some bands of these data are heavily contaminated by noise and cannot be regarded as clean ground truth, and thus have to be deleted. The color composite representations of original HSIs are shown in Fig.4. The simulation strategy is the same as that in the previous section, but with varying noise intensity of $0.02,0.04,0.06$, $0.08,0.1,0.2$, and 0.3 . The means of MPSNR, MSSIM and time at different scenes and noise levels are presented in Table 1 , and the detailed results are provided in Tables 4-7 in Appendix A. Dealing with different levels of noise under different scenes, LRCOD yields the best MPSNR performance in 30 of 35 cases (Table 4). Although LECOD performs less well in MSSIM than FastHyDe and SNLRSF, it is competitive in comparison with BM4D and SSTV. Moreover, without searching for similar patches LRCOD runs faster than other five methods, although slower than FastHyDe since the latter is a non-iterative algorithm.

Some denoising results are shown in Fig.5. Our method can obtain competitive recovery performance in comparison with others. High quality spectral signature is of critical importance to material identification. The quality of reconstructed spectra from different denoising methods can also be inferred from Fig.6 and Fig.7. Clearly, LRCOD can recover spectral signatures better than others in many cases. Even with noise level 0.1 for Japser Ridge, our method can restore spectral signatures better than others, especially between bands 150 and 200 .

Now we compare the MPSNRs and computing time of LRCOD and FS ${ }^{2}$ LRL [37]. From Table 2, we can observe that: the performance of FS ${ }^{2} L R L$ improves slightly as the number of superpixels increases, and FS ${ }^{2}$ LRL at 200 superpixels is competitive with LRCOD. However, having more 


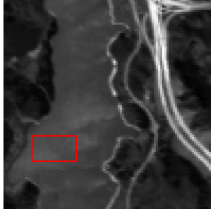

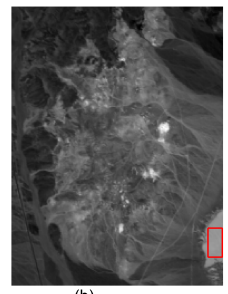

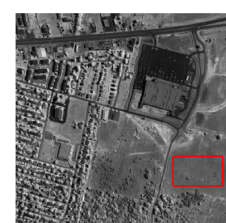

(c)

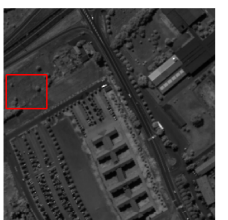

(d)

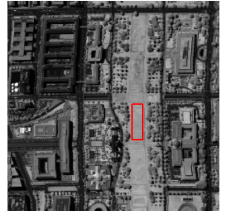

(e)

Figure 4: The representations of HSls: (a) Japser Ridge, (b) Cuprite, (c) Urban, (d) Pavia, (e) Washington DC Mall. The band 10 is used for (a) while band 76 is used for (b)-(e). The red boxes point out the homogeneous regions selected for the computation of the ENL metric.

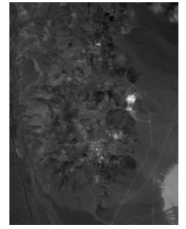

(a)

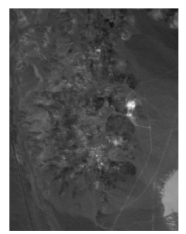

(f)

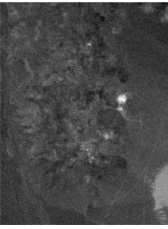

(b)

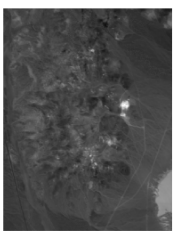

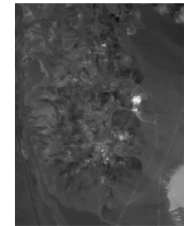

(c)

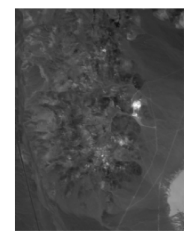

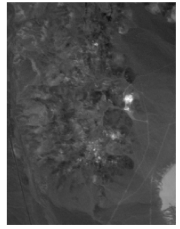

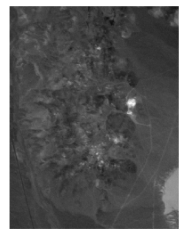

(d)

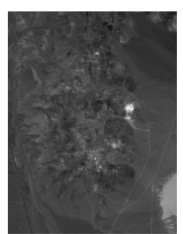

(i)

Figure 5: Denoised results by different methods on band 140 of Cuprite. (a) Clean image. (b) Noisy image. Recovered images by (c) BM4D, (d) LRMR, (e) SSTV, (f) NMoG, (g) FastHyDe, (h) SNLRSF, (i) LRCOD.
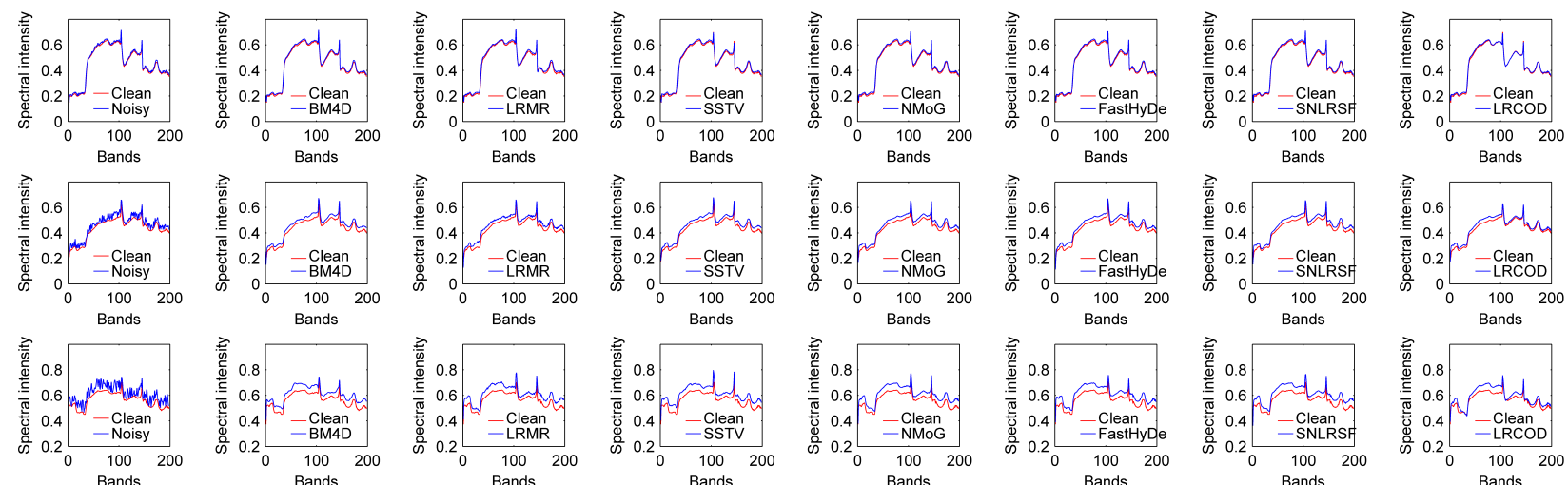

Figure 6: Denoised spectral signatures of Japser Ridge with noise levels 0.02 (first row), 0.06 (second row), and 0.1 (third row).

Table 1

The means of MPSNR, MSSIM, running time, and rel.ENL of different algorithms at 35 cases. The detailed results for all 35 cases are provided in Tables 4-7 in Appendix A.

\begin{tabular}{cccccccc}
\hline Index & BM4D & LRMR & SSTV & NMoG & FastHyDe & SNLRSF & LRCOD \\
\hline MPSNR & 27.6775 & 27.6998 & 27.5055 & 27.4680 & 27.7212 & 27.4651 & 27.9194 \\
MSSIM & 0.9011 & 0.9046 & 0.8899 & 0.9050 & 0.9108 & 0.9104 & 0.9034 \\
Time & 418.85 & 51.56 & 378.25 & 170.68 & 0.55 & 57.94 & 23.39 \\
rel.ENL & 0.5652 & 0.2809 & 0.2161 & 0.2461 & 0.4947 & 0.4164 & 0.2304 \\
\hline
\end{tabular}


Table 2

MPSNRs and computing time (seconds) of LRCOD and FS 2 LRL, where scenes 1-5 are from Japser Ridge, Cuprite, Urban, Pavia Centre and Washington DC Mall, respectively. Cases 1-7 correspond to noise levels $0.02,0.04,0.06,0.08,0.1,0.2$ and 0.3 , respectively. In FS'LRL, 20, 100 and 200 superpixels are applied, respectively.

\begin{tabular}{|c|c|c|c|c|c|c|c|c|}
\hline \multirow{3}{*}{ Method } & \multicolumn{4}{|c|}{ MPSNRs } & \multicolumn{4}{|c|}{ Time } \\
\hline & \multicolumn{3}{|c|}{$\mathrm{FS}^{2} \mathrm{LRL}$} & \multirow[t]{2}{*}{ LRCOD } & \multicolumn{3}{|c|}{$\mathrm{FS}^{2} \mathrm{LRL}$} & \multirow[t]{2}{*}{ LRCOD } \\
\hline & 20 & 100 & 200 & & 20 & 100 & 200 & \\
\hline scene 1 , case 1 & 37.55 & 37.61 & 37.66 & 38.21 & 22.03 & 96.49 & 190.57 & 7.27 \\
\hline scene 1 , case 2 & 33.47 & 33.55 & 33.62 & 33.90 & 21.67 & 96.43 & 190.37 & 6.76 \\
\hline scene 1 , case 3 & 30.37 & 30.45 & 30.50 & 30.80 & 21.92 & 96.82 & 190.34 & 7.00 \\
\hline scene 1 , case 4 & 28.03 & 28.08 & 28.12 & 28.41 & 21.85 & 97.86 & 192.22 & 5.63 \\
\hline scene 1 , case 5 & 26.17 & 26.23 & 26.27 & 26.57 & 22.18 & 97.51 & 191.57 & 2.64 \\
\hline scene 1 , case 6 & 20.37 & 20.43 & 20.47 & 20.53 & 21.83 & 96.90 & 189.66 & 2.30 \\
\hline scene 1 , case 7 & 16.92 & 16.98 & 17.02 & 16.89 & 21.72 & 96.13 & 190.63 & 2.07 \\
\hline scene 2 , case 1 & 38.76 & 38.87 & 38.92 & 38.48 & 91.04 & 402.44 & 791.35 & 39.37 \\
\hline scene 2 , case 2 & 34.06 & 34.13 & 34.18 & 34.19 & 90.90 & 405.24 & 799.21 & 24.24 \\
\hline scene 2 , case 3 & 30.70 & 30.76 & 30.79 & 30.85 & 92.33 & 410.29 & 800.55 & 18.72 \\
\hline scene 2 , case 4 & 28.21 & 28.24 & 28.28 & 28.28 & 92.53 & 430.41 & 845.75 & 15.87 \\
\hline scene 2 , case 5 & 26.27 & 26.30 & 26.32 & 26.24 & 97.36 & 432.85 & 848.53 & 16.72 \\
\hline scene 2 , case 6 & 20.20 & 20.21 & 20.22 & 20.25 & 97.18 & 404.17 & 793.50 & 22.45 \\
\hline scene 2 , case 7 & 16.69 & 16.71 & 16.72 & 16.69 & 90.80 & 403.13 & 800.19 & 21.21 \\
\hline scene 3 , case 1 & 38.41 & 38.46 & 38.49 & 39.56 & 154.46 & 685.43 & 1346.14 & 122.85 \\
\hline scene 3 , case 2 & 34.05 & 34.09 & 34.12 & 34.06 & 154.75 & 704.86 & 1422.73 & 48.73 \\
\hline scene 3 , case 3 & 30.75 & 30.79 & 30.82 & 30.63 & 161.66 & 722.94 & 1432.74 & 32.96 \\
\hline scene 3 , case 4 & 28.28 & 28.31 & 28.33 & 28.17 & 163.16 & 726.90 & 1426.95 & 57.96 \\
\hline scene 3 , case 5 & 26.32 & 26.35 & 26.37 & 26.22 & 163.20 & 725.00 & 1420.35 & 15.91 \\
\hline scene 3 , case 6 & 20.19 & 20.21 & 20.22 & 20.21 & 161.69 & 722.98 & 1426.79 & 44.03 \\
\hline scene 3 , case 7 & 16.63 & 16.64 & 16.64 & 16.61 & 161.49 & 722.18 & 1426.62 & 16.96 \\
\hline scene 4 , case 1 & 38.56 & 38.66 & 38.76 & 38.76 & 76.74 & 341.53 & 670.43 & 18.71 \\
\hline scene 4 , case 2 & 34.30 & 34.39 & 34.46 & 34.25 & 76.43 & 340.40 & 671.86 & 25.68 \\
\hline scene 4 , case 3 & 30.96 & 31.02 & 31.07 & 31.02 & 76.44 & 342.16 & 674.31 & 6.84 \\
\hline scene 4 , case 4 & 28.45 & 28.50 & 28.53 & 28.54 & 76.62 & 342.21 & 672.87 & 7.19 \\
\hline scene 4 , case 5 & 26.47 & 26.50 & 26.53 & 26.56 & 76.70 & 343.12 & 675.09 & 6.24 \\
\hline scene 4 , case 6 & 20.27 & 20.29 & 20.30 & 20.34 & 76.58 & 341.88 & 672.11 & 10.10 \\
\hline scene 4 , case 7 & 16.71 & 16.73 & 16.74 & 16.71 & 76.51 & 341.15 & 674.38 & 10.56 \\
\hline scene 5 , case 1 & 37.85 & 37.88 & 37.90 & 39.07 & 143.69 & 646.53 & 1275.23 & 82.38 \\
\hline scene 5 , case 2 & 33.78 & 33.82 & 33.85 & 33.84 & 144.66 & 647.16 & 1274.82 & 17.81 \\
\hline scene 5 , case 3 & 30.61 & 30.65 & 30.67 & 30.73 & 145.72 & 647.81 & 1275.51 & 17.75 \\
\hline scene 5 , case 4 & 28.20 & 28.22 & 28.24 & 28.31 & 145.21 & 651.83 & 1279.88 & 15.42 \\
\hline scene 5 , case 5 & 26.26 & 26.29 & 26.30 & 26.38 & 144.91 & 648.68 & 1268.42 & 18.85 \\
\hline scene 5 , case 6 & 20.18 & 20.19 & 20.20 & 20.24 & 143.70 & 642.38 & 1266.55 & 29.23 \\
\hline scene 5 , case 7 & 16.64 & 16.65 & 16.66 & 16.65 & 143.95 & 643.21 & 1275.23 & 20.31 \\
\hline mean & 27.76 & 27.80 & 27.84 & 27.92 & 99.25 & 442.77 & 872.67 & 23.39 \\
\hline
\end{tabular}

superpixels means computationally more costly: FS ${ }^{2} \mathrm{LRL}$ needs 873 seconds on average while LRCOD 23 seconds.

Furthermore, we evaluate the degree of smoothing in an image region $\mathbf{S}$ by computing the equivalent number of looks (ENL) [46], which is defined as

$$
\operatorname{ENL}(\mathbf{S})=\frac{\mu^{2}}{\sigma^{2}}
$$

where $\mu$ and $\sigma$ are the mean and standard deviation of $\mathbf{S}$ respectively. When the clean image region is available, we can evaluate how approximate it is between the ENL of clean region and that of recovered region by using relative ENL error. Specifically, denote the clean, observed, and recovered image regions as $\mathbf{S}^{c l n}, \mathbf{S}^{\text {obr }}$, and $\mathbf{S}^{r e c}$, where $\mathbf{S}^{r e c}$ is recovered from $\mathbf{S}^{o b r}$, then the relative ENL error 'rel.ENL' between $\mathbf{S}^{c l n}$ and $\mathbf{S}^{r e c}$ can be defined by

$$
\operatorname{rel.ENL}\left(\mathbf{S}^{c l n}, \mathbf{S}^{r e c}\right)=\frac{\left|\operatorname{ENL}\left(\mathbf{S}^{r e c}\right)-\operatorname{ENL}\left(\mathbf{S}^{c l n}\right)\right|}{\operatorname{ENL}\left(\mathbf{S}^{c l n}\right)}
$$

The quantitative improvements of LRCOD can be reflected by the numerical results. As shown in Table 1, LRCOD shows a mean gain of about $0.335,0.051,0.016,0.264$ and 0.186 with respect to BM4D, LRMR, NMoG, FastHyDe, and SNLRSF respectively. These improvements testify a better image restoration capability of LRCOD compared with other methods. However, LRCOD underperforms the SSTV 

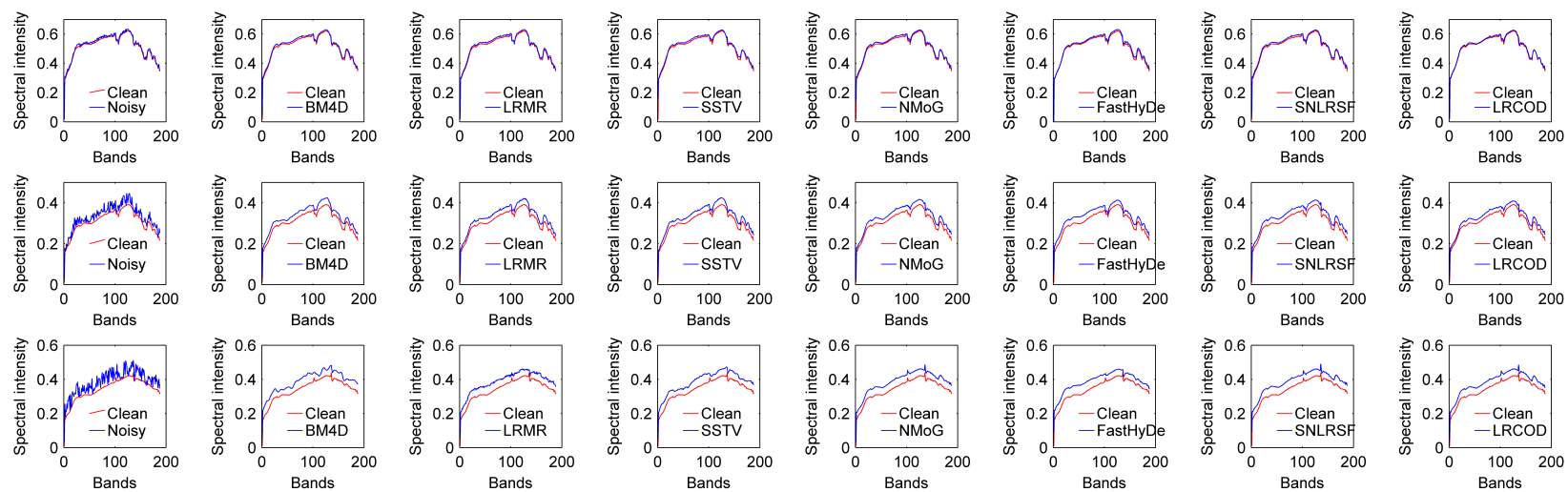

Figure 7: Denoised spectral signatures of Cuprite data with noise levels 0.02 (first row), 0.06 (second row), and 0.1 (third row).

Table 3

The ENLs on selected homogeneous regions from Indian Pines (second row) and Urban (third row).

\begin{tabular}{cccccccc}
\hline Observation & BM4D & LRMR & SSTV & NMoG & FastHyDe & SNLRSF & LRCOD \\
\hline 15.8398 & 26.0048 & 174.8623 & 291.5271 & 536.4398 & 424.2250 & 583.9305 & 700.7684 \\
8.7465 & 23.8963 & 21.6304 & 103.7517 & 13.9542 & 65.2848 & 18.5470 & 65.8369 \\
\hline
\end{tabular}

method, since SSTV can enhance smoothness by utilizing total variation along the spatial dimension.

\subsection{Experiments on real data}

Two real HSI datasets are adopted to further demonstrate the denoising performance of LRCOD. One is the Urban dataset of size $307 \times 307 \times 210$, whose bands $1-4,76,87,101$ $111,136-153$ and 198-210 are polluted due to dense water vapor and atmospheric effects. Another dataset is the Indian Pines dataset of size $145 \times 145 \times 220$, some of whose bands are polluted by a mixture of Gaussian noise, impulse noise, and water absorption.

Denoising results from different methods are given in Fig. 8 and Fig.9. It can be clearly observed that the methods LRMR, SSTV, and NMoG perform less well than our LRCOD. The FastHyDe and SNLRSF achieve competitive visual quality compared to LRCOD, but cause the restoration results to be over smooth, as Fig.8(f) and Fig.8(g) show. In Fig.9, both LRCOD and SNLRSF outperform others, but SNLRSF's time consumption is larger since it needs to seek the nonlocal similar patches to form a tensor. Table 3 shows the numerical results of ENLs, where LRCOD performs best on Indian Pines while underperforming SSTV on Urban.

\section{Conclusion and Future Work}

This work proposed a novel HSI denoising model called LRCOD. In LRCOD, the low rankness of representation coefficients is exploited, opening a new perspective for HSI denoising; furthermore, an orthonormal dictionary is learned, generalizing the fixed-dictionary based denoising models.

In future, one can generalize the representation model
(2) into a three components model, that is, to represent $\mathbf{Y}$ by $\mathbf{Y}=\mathbf{E Z M}+\mathbf{N}$, where $\mathbf{E}$ and $\mathbf{M}$ are selected to decorrelate the signal and noise in the HSI spatially and spectrally, respectively, and they can be known or unknown. As a result, LRCOD can be generalized by adding the matrix $\mathbf{M}$ and its corresponding constraint. In addition, in LRCOD we made no assumption for noise types. We can modify LRCOD to focus on specific and challenging noise, such as non-i.i.d. noise.

\section{Declaration of Competing Interest}

None.

\section{CRediT authorship contribution statement}

Fanlong Zhang: Conceptualization, Methodology, Software, Writing - original draft. Guowei Yang: Project administration, Funding acquisition. Jing-Hao Xue: Writing - review \& editing, Supervision.

\section{Acknowledgements}

We thank the reviewers for their constructive suggestions that improved our work. This work was partly supported by the National Natural Science Foundation of China (Grant Nos. 61603192, U1831127, and 61876213), Jiangsu Government Scholarship for Overseas Studies under Grant No. JS-2018-068, and National Key Research and Development Program of China under Grant No. 2017YFC0804002. 
Hyperspectral image denoising based on low-rank coefficients and orthonormal dictionary

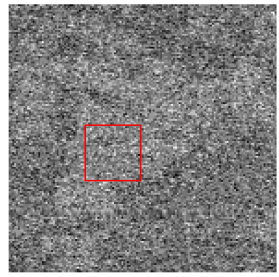

(a)

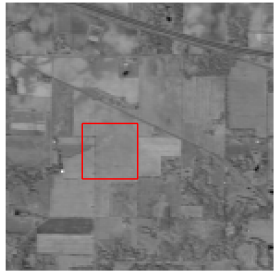

(e)

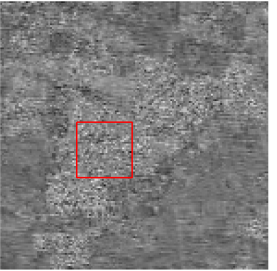

(b)

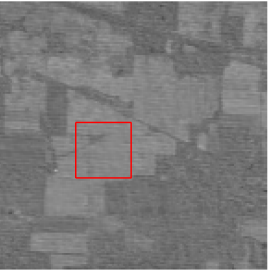

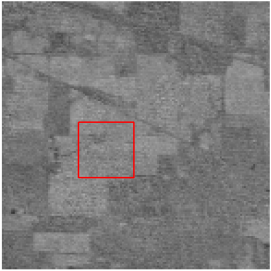

(c)

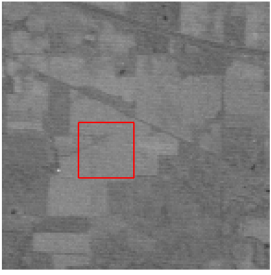

(g)
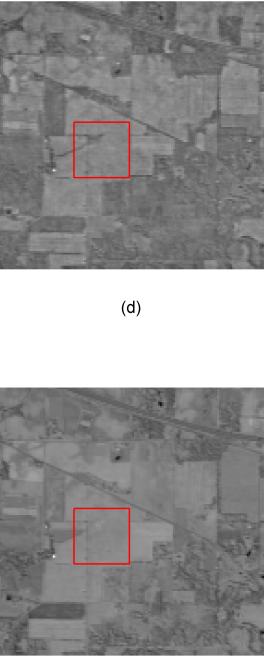

Figure 8: Denoising results in Indian Pines. (a) Observation; (b) BM4D; (c) LRMR; (d) SSTV; (e) NMoG; (f) FastHyDe; (g) SNLRSF; (h) LRCOD. The red boxes point out the homogeneous regions selected for the computation of the ENL metric.

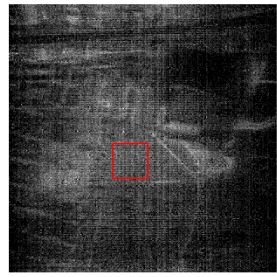

(a)

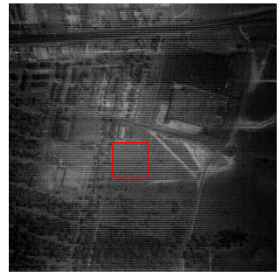

(e)

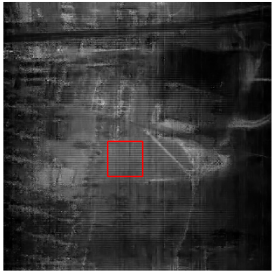

(b)

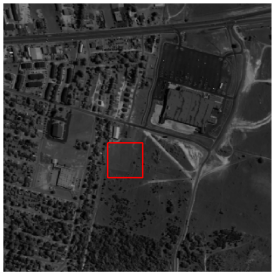

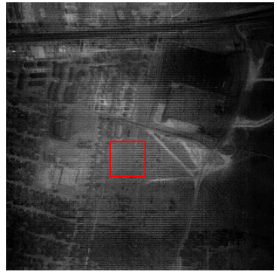

(c)

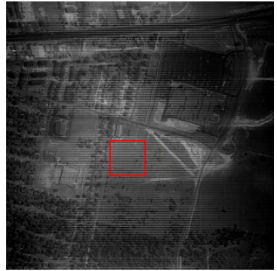

(g)
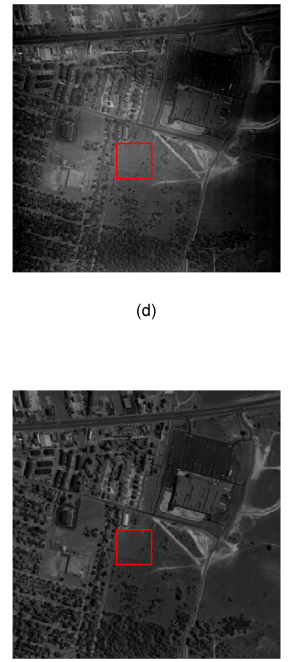

Figure 9: Denoising results in Urban. (a) Observation; (b) BM4D; (c) LRMR; (d) SSTV; (e) NMoG; (f) FastHyDe; (g) SNLRSF; (h) LRCOD. The red boxes point out the homogeneous regions selected for the computation of the ENL metric.

\section{References}

[1] U. B. Gewali, S. T. Monteiro, E. Saber, Machine learning based hyperspectral image analysis: A survey, arXiv preprint arXiv:1802.08701 (2018).

[2] G. Camps-Valls, D. Tuia, L. Bruzzone, J. A. Benediktsson, Advances in hyperspectral image classification: Earth monitoring with statistical learning methods, IEEE Signal Processing Magazine 31 (1) (2014) 45-54.

[3] Z. Wang, J. Liu, J.-H. Xue, Joint sparse model-based discriminative K-SVD for hyperspectral image classification, Signal Processing 133 (2017) 144-155

[4] Z. Wang, R. Zhu, K. Fukui, J.-H. Xue, Cone-based joint sparse modelling for hyperspectral image classification, Signal Processing 144 (2018) 417-429.

[5] X. Cao, J. Yao, Z. Xu, D. Meng, Hyperspectral image classification with convolutional neural network and active learning, IEEE Transactions on Geoscience and Remote Sensing 58 (7) (2020) 4604-4616.

[6] Q. Liu, L. Xiao, J. Yang, J. C.-W. Chan, Content-guided convolutional neural network for hyperspectral image classification, IEEE Transactions on Geoscience and Remote Sensing (2020).

[7] J. Zhao, S. Tian, C. Geiß, L. Wang, Y. Zhong, H. Taubenböck, Spectral-spatial classification integrating band selection for hyperspectral imagery with severe noise bands, IEEE Journal of Selected Topics in Applied Earth Observations and Remote Sensing 13 (2020) 1597-1609.

[8] N. M. Nasrabadi, Hyperspectral target detection: An overview of current and future challenges, IEEE Signal Processing Magazine 31 (1) (2014) 34-44.

[9] Z. Wang, R. Zhu, K. Fukui, J.-H. Xue, Matched shrunken cone detector (MSCD): Bayesian derivations and case studies for hyperspectral target detection, IEEE Transactions on Image Processing 26 (11) 
(2017) 5447-5461.

[10] X. Yang, L. Zhang, L. Gao, J.-H. Xue, MSDH: Matched subspace detector with heterogeneous noise, Pattern Recognition Letters 125 (2019) 701-707.

[11] X. Yang, M. Dong, Z. Wang, L. Gao, L. Zhang, J.-H. Xue, Dataaugmented matched subspace detector for hyperspectral subpixel target detection, Pattern Recognition (2020) 107464.

[12] R. Feng, L. Wang, Y. Zhong, Joint local block grouping with noiseadjusted principal component analysis for hyperspectral remotesensing imagery sparse unmixing, Remote Sensing 11 (10) (2019) 1223.

[13] L. Drumetz, T. R. Meyer, J. Chanussot, A. L. Bertozzi, C. Jutten, Hyperspectral image unmixing with endmember bundles and group sparsity inducing mixed norms, IEEE Transactions on Image Processing 28 (7) (2019) 3435-3450.

[14] L. Sun, F. Wu, T. Zhan, W. Liu, J. Wang, B. Jeon, Weighted nonlocal low-rank tensor decomposition method for sparse unmixing of hyperspectral images, IEEE Journal of Selected Topics in Applied Earth Observations and Remote Sensing 13 (2020) 1174-1188.

[15] H. Li, R. Feng, L. Wang, Y. Zhong, L. Zhang, Superpixel-based reweighted low-rank and total variation sparse unmixing for hyperspectral remote sensing imagery, IEEE Transactions on Geoscience and Remote Sensing (2020).

[16] B. Rasti, P. Scheunders, P. Ghamisi, G. Licciardi, J. Chanussot, Noise reduction in hyperspectral imagery: Overview and application, Remote Sensing 10 (3) (2018) 482

[17] K. Dabov, A. Foi, V. Katkovnik, K. Egiazarian, Image denoising by sparse 3-D transform-domain collaborative filtering, IEEE Transactions on Image Processing 16 (8) (2007) 2080-2095.

[18] Z. Kong, X. Yang, Color image and multispectral image denoising using block diagonal representation, IEEE Transactions on Image Processing 28 (9) (2019) 4247-4259.

[19] E. Vargas, H. Arguello, J.-Y. Tourneret, Spectral image fusion from compressive measurements using spectral unmixing and a sparse representation of abundance maps, IEEE Transactions on Geoscience and Remote Sensing 57 (7) (2019) 5043-5053.

[20] L. Zhuang, J. M. Bioucas-Dias, Fast hyperspectral image denoising and inpainting based on low-rank and sparse representations, IEEE Journal of Selected Topics in Applied Earth Observations and Remote Sensing 11 (3) (2018) 730-742.

[21] C. Cao, J. Yu, C. Zhou, K. Hu, F. Xiao, X. Gao, Hyperspectral image denoising via subspace-based nonlocal low-rank and sparse factorization, IEEE Journal of Selected Topics in Applied Earth Observations and Remote Sensing 12 (3) (2019) 973-988.

[22] H. Zhang, W. He, L. Zhang, H. Shen, Q. Yuan, Hyperspectral image restoration using low-rank matrix recovery, IEEE Transactions on Geoscience and Remote Sensing 52 (8) (2014) 4729-4743.

[23] H. Fan, J. Li, Q. Yuan, X. Liu, M. Ng, Hyperspectral image denoising with bilinear low rank matrix factorization, Signal Processing 163 (2019) 132-152.

[24] B. Du, Z. Huang, N. Wang, A bandwise noise model combined with low-rank matrix factorization for hyperspectral image denoising, IEEE Journal of Selected Topics in Applied Earth Observations and Remote Sensing 11 (4) (2018) 1070-1081.

[25] Y. Peng, D. Meng, Z. Xu, C. Gao, Y. Yang, B. Zhang, Decomposable nonlocal tensor dictionary learning for multispectral image denoising, in: Proceedings of the IEEE Conference on Computer Vision and Pattern Recognition, 2014, pp. 2949-2956.

[26] Q. Xie, Q. Zhao, D. Meng, Z. Xu, Kronecker-basis-representation based tensor sparsity and its applications to tensor recovery, IEEE Transactions on Pattern Analysis and Machine Intelligence 40 (8) (2017) 1888-1902.

[27] X. Gong, W. Chen, J. Chen, A low-rank tensor dictionary learning method for hyperspectral image denoising, IEEE Transactions on Signal Processing 68 (2020) 1168-1180.

[28] X. Zhang, C. Li, J. Zhang, Q. Chen, J. Feng, L. Jiao, H. Zhou, Hyperspectral unmixing via low-rank representation with space consistency constraint and spectral library pruning, Remote Sensing 10 (2) (2018)
339.

[29] Y. Ma, Q. Jin, X. Mei, X. Dai, F. Fan, H. Li, J. Huang, Hyperspectral unmixing with Gaussian mixture model and low-rank representation, Remote Sensing 11 (8) (2019) 911.

[30] Q. Yuan, Q. Zhang, J. Li, H. Shen, L. Zhang, Hyperspectral image denoising employing a spatial-spectral deep residual convolutional neural network, IEEE Transactions on Geoscience and Remote Sensing 57 (2) (2018) 1205-1218.

[31] K. Wei, Y. Fu, H. Huang, 3-d quasi-recurrent neural network for hyperspectral image denoising, IEEE Transactions on Neural Networks and Learning Systems (2020).

[32] J. M. Bioucas-Dias, A. Plaza, N. Dobigeon, M. Parente, Q. Du, P. Gader, J. Chanussot, Hyperspectral unmixing overview: Geometrical, statistical, and sparse regression-based approaches, IEEE Journal of Selected Topics in Applied Earth Observations and Remote Sensing 5 (2) (2012) 354-379.

[33] M.-D. Iordache, J. M. Bioucas-Dias, A. Plaza, Sparse unmixing of hyperspectral data, IEEE Transactions on Geoscience and Remote Sensing 49 (6) (2011) 2014-2039.

[34] Q. Qu, N. M. Nasrabadi, T. D. Tran, Abundance estimation for bilinear mixture models via joint sparse and low-rank representation, IEEE Transactions on Geoscience and Remote Sensing 52 (7) (2013) 4404-4423.

[35] R. A. Horn, C. R. Johnson, Matrix analysis, Cambridge university press, 2012.

[36] J. M. Bioucas-Dias, J. M. Nascimento, Hyperspectral subspace identification, IEEE Transactions on Geoscience and Remote Sensing 46 (8) (2008) 2435-2445.

[37] L. Sun, B. Jeon, B. N. Soomro, Y. Zheng, Z. Wu, L. Xiao, Fast superpixel based subspace low rank learning method for hyperspectral denoising, IEEE Access 6 (2018) 12031-12043.

[38] P. Jain, P. Kar, Non-convex optimization for machine learning, Foundations and Trends $®$ in Machine Learning 10 (3-4) (2017) 142-336.

[39] Y. Chi, Y. M. Lu, Y. Chen, Nonconvex optimization meets low-rank matrix factorization: An overview, IEEE Transactions on Signal Processing 67 (20) (2019) 5239-5269.

[40] J.-F. Cai, E. J. Candès, Z. Shen, A singular value thresholding algorithm for matrix completion, SIAM Journal on optimization 20 (4) (2010) 1956-1982.

[41] Z. Wen, W. Yin, A feasible method for optimization with orthogonality constraints, Mathematical Programming 142 (1-2) (2013) 397434.

[42] Y. Chen, X. Cao, Q. Zhao, D. Meng, Z. Xu, Denoising hyperspectral image with non-iid noise structure, IEEE Transactions on Cybernetics 48 (3) (2018) 1054-1066.

[43] M. Maggioni, V. Katkovnik, K. Egiazarian, A. Foi, Nonlocal transform-domain filter for volumetric data denoising and reconstruction, IEEE Transactions on Image Processing 22 (1) (2013) 119-133.

[44] H. K. Aggarwal, A. Majumdar, Hyperspectral image denoising using spatio-spectral total variation, IEEE Geoscience and Remote Sensing Letters 13 (3) (2016) 442-446.

[45] Q. Li, Z. Zhu, G. Tang, Alternating minimizations converge to second-order optimal solutions, in: International Conference on Machine Learning, 2019, pp. 3935-3943.

[46] F. Lattari, B. Gonzalez Leon, F. Asaro, A. Rucci, C. Prati, M. Matteucci, Deep learning for SAR image despeckling, Remote Sensing 11 (13) (2019) 1532.

\section{A. Appendix}


Hyperspectral image denoising based on low-rank coefficients and orthonormal dictionary

Table 4

MPSNRs of different algorithms, where scenes 1-5 are from Japser Ridge, Cuprite, Urban, Pavia Centre and Washington DC Mall, respectively. Cases 1-7 correspond to noise levels $0.02,0.04,0.06,0.08,0.1,0.2$ and 0.3 , respectively. The mean performances are in bold.

\begin{tabular}{|c|c|c|c|c|c|c|c|}
\hline Cases & BM4D & LRMR & SSTV & NMoG & FastHyDe & SNLRSF & LRCOD \\
\hline scene 1 , Case 1 & & & & & & & \\
\hline scene 1 , Case 2 & 33.7401 & 337 & 33.4377 & & & & 8975 \\
\hline scene 1 , Case 3 & 30.2685 & 30.2794 & 30.1174 & & & & 7987 \\
\hline ene 1 ,Case 4 & 27.8022 & 27.8175 & 27.7104 & 27.6965 & 27.8788 & 7078 & 4122 \\
\hline scene 1 , Case 5 & 25.8812 & 25.8896 & 25.8118 & 25.8270 & 25.9496 & 25.8360 & 26.5720 \\
\hline scene 1 , Case 6 & 19.9087 & 19.9062 & 19.8366 & 19.9193 & 62 & 19.9298 & 20.5308 \\
\hline scene 1 , Case 7 & 16.4103 & 16.4 & 16.3393 & & & 413 & 5.8937 \\
\hline ne 2 ,Case 1 & 39.6 & 39.7 & 39.4 & & & & 787 \\
\hline e 2 & 33. & 33. & 33. & & & & 946 \\
\hline scene 2 & 30.2 & 30. & 64 & & & & 533 \\
\hline se 4 & 27.8 & 27. & 55 & & & & 2805 \\
\hline se 5 & 25.8826 & 25.9319 & 25.8424 & 25.8740 & 8727 & 330 & 26.2353 \\
\hline scene 2, Case 6 & 19.9119 & 19. & 103 & & & & 20.2539 \\
\hline scene 2 , Case 7 & 16.4 & 16. & & & & & \\
\hline scene 3 , Case 1 & 39.6 & 39.7 & 38.7 & $3 \varepsilon$ & & & 96 \\
\hline e 3 , Case 2 & 33.7 & 33. & & & & & 99 \\
\hline e 3 & 30.2 & 30. & 92 & & & & 29 \\
\hline 4 & 27.7 & $27 \varepsilon r-r \cdot r$ & 00 & & & & 679 \\
\hline scene 3 , Case 5 & 25.8790 & & & & & & 26.2201 \\
\hline scene 3 , Case 6 & 19.9038 & 19.9392 & 19.8561 & & & & 20.2133 \\
\hline scene 3 , Case 7 & 16.3996 & 16.4208 & 16.3440 & & & & 16.6111 \\
\hline scene 4 , Case 1 & 39.7119 & 39.6700 & 39.3779 & 38.8 & & 38 & 38. \\
\hline scene 4 , Case 2 & 33.7759 & 33.7766 & 33.7243 & & & & 44 \\
\hline se 3 & 30.2 & 30. & 30. & 30 & 13 & & 31 \\
\hline scene 4 , & 27.8 & 27.8 & 54 & 27. & 75 & 84 & 443 \\
\hline scene 4 , Case 5 & 25.9162 & 25.8993 & 25.9006 & 25.9005 & 17 & 84 & 26.5629 \\
\hline scene 4 , Case 6 & 19.9437 & 19.9124 & 19.8741 & 19.9395 & 19.9583 & 19.9609 & 20.3395 \\
\hline scene 4 , Case 7 & 16.4388 & 16.4002 & 16.3566 & 16.4358 & 16.4500 & 16.4546 & 16.7124 \\
\hline scene 5 , Case 1 & 39.6629 & 39.7313 & 38.6044 & 38.7508 & 39.9367 & 38.6397 & 39.0700 \\
\hline scene 5 , Case 2 & 33.7530 & 33.8230 & 33.4520 & & & & 33.8382 \\
\hline scene 5 , Case 3 & 30.2782 & 30.3 & 30.1 & & & & 30. \\
\hline scene 5 , Case 4 & 27.8092 & 27.8625 & 27.7476 & 27.8220 & 27.9151 & 27.8237 & 28.3114 \\
\hline scene 5 , Case 5 & 25.8902 & 25.9393 & 25.8493 & 25.9230 & 25.9814 & 25.9284 & 26.3752 \\
\hline scene 5 , Case 6 & 19.9130 & 19.9407 & 19.8581 & 19.9568 & 19.9689 & 19.9643 & 20.2422 \\
\hline scene 5 , Case 7 & 16.4111 & 16.4230 & 16.3495 & 16.4498 & 16.4540 & 16.4564 & 16.6499 \\
\hline mean & 27.6775 & 27.6998 & 27.5055 & 27.4680 & 27.7212 & 27.4651 & 27.9194 \\
\hline
\end{tabular}


Hyperspectral image denoising based on low-rank coefficients and orthonormal dictionary

Table 5

MSSIMs of different algorithms, where scenes 1-5 are from Japser Ridge, Cuprite, Urban, Pavia Centre and Washington DC Mall, respectively. Cases 1-7 correspond to noise levels $0.02,0.04,0.06,0.08,0.1,0.2$ and 0.3 , respectively. The mean performances are in bold.

\begin{tabular}{|c|c|c|c|c|c|c|c|}
\hline Cases & BM4D & LRMR & SSTV & NMoG & FastHyDe & SNLRSF & LRCOD \\
\hline scene 1 , Case 1 & 0.9815 & 0.9817 & 0.9738 & 0.9732 & 0.9809 & 0.9741 & 0.9701 \\
\hline scene 1, Case 2 & 0.9512 & 0.9528 & 0.9450 & 0.9460 & 0.9518 & 0.9466 & 0.9429 \\
\hline scene 1 , Case 3 & 0.9211 & 0.9234 & 0.9157 & 0.9180 & 0.9236 & 0.9190 & 0.9123 \\
\hline scene 1 , Case 4 & 0.8936 & 0.8964 & 0.8882 & 0.8921 & 0.8980 & 0.8938 & 0.8797 \\
\hline scene 1 , Case 5 & 0.8685 & 0.8715 & 0.8624 & 0.8684 & 0.8748 & 0.8710 & 0.8541 \\
\hline scene 1 , Case 6 & 0.7705 & 0.7735 & 0.7474 & 0.7751 & 0.7857 & 0.7839 & 0.7694 \\
\hline scene 1, Case 7 & 0.6970 & 0.6991 & 0.6594 & 0.7067 & 0.7214 & 0.7211 & 0.7057 \\
\hline scene 2 , Case 1 & 0.9954 & 0.9964 & 0.9913 & 0.9865 & 0.9869 & 0.9896 & 0.9888 \\
\hline scene 2 , Case 2 & 0.9865 & 0.9900 & 0.9855 & 0.9821 & 0.9780 & 0.9850 & 0.9797 \\
\hline scene 2 , Case 3 & 0.9755 & 0.9815 & 0.9769 & 0.9765 & 0.9712 & 0.9787 & 0.9747 \\
\hline scene 2 , Case 4 & 0.9634 & 0.9718 & 0.9660 & 0.9683 & 0.9641 & 0.9712 & 0.9679 \\
\hline scene 2 , Case 5 & 0.9501 & 0.9607 & 0.9525 & 0.9589 & 0.9559 & 0.9626 & 0.9593 \\
\hline scene 2 , Case 6 & 0.8776 & 0.8942 & 0.8508 & 0.9019 & 0.9068 & 0.9118 & 0.9054 \\
\hline scene 2 , Case 7 & 0.8042 & 0.8176 & 0.7417 & 0.8376 & 0.8521 & 0.8557 & 0.8428 \\
\hline scene 3, Case 1 & 0.9923 & 0.9932 & 0.9881 & 0.9912 & 0.9937 & 0.9913 & 0.9917 \\
\hline scene 3, Case 2 & 0.9766 & 0.9792 & 0.9741 & 0.9779 & 0.9803 & 0.9783 & 0.9749 \\
\hline scene 3, Case 3 & 0.9574 & 0.9619 & 0.9562 & 0.9611 & 0.9637 & 0.9622 & 0.9597 \\
\hline scene 3 , Case 4 & 0.9367 & 0.9428 & 0.9361 & 0.9424 & 0.9455 & 0.9445 & 0.9419 \\
\hline scene 3, Case 5 & 0.9153 & 0.9229 & 0.9145 & 0.9230 & 0.9268 & 0.9261 & 0.9209 \\
\hline scene 3 , Case 6 & 0.8113 & 0.8227 & 0.7922 & 0.8262 & 0.8353 & 0.8360 & 0.8294 \\
\hline scene 3, Case 7 & 0.7201 & 0.7307 & 0.6833 & 0.7402 & 0.7552 & 0.7566 & 0.7421 \\
\hline scene 4, Case 1 & 0.9929 & 0.9933 & 0.9887 & 0.9855 & 0.9947 & 0.9883 & 0.9851 \\
\hline scene 4, Case 2 & 0.9783 & 0.9800 & 0.9769 & 0.9755 & 0.9811 & 0.9773 & 0.9716 \\
\hline scene 4, Case 3 & 0.9604 & 0.9624 & 0.9607 & 0.9603 & 0.9640 & 0.9626 & 0.9526 \\
\hline scene 4, Case 4 & 0.9413 & 0.9426 & 0.9415 & 0.9423 & 0.9461 & 0.9461 & 0.9368 \\
\hline scene 4, Case 5 & 0.9216 & 0.9215 & 0.9197 & 0.9227 & 0.9274 & 0.9285 & 0.9192 \\
\hline scene 4, Case 6 & 0.8217 & 0.8091 & 0.7863 & 0.8182 & 0.8349 & 0.8376 & 0.8221 \\
\hline scene 4, Case 7 & 0.7302 & 0.7003 & 0.6620 & 0.7206 & 0.7518 & 0.7538 & 0.7249 \\
\hline scene 5, Case 1 & 0.9919 & 0.9924 & 0.9879 & 0.9892 & 0.9932 & 0.9890 & 0.9886 \\
\hline scene 5 , Case 2 & 0.9750 & 0.9767 & 0.9725 & 0.9745 & 0.9781 & 0.9744 & 0.9704 \\
\hline scene 5 , Case 3 & 0.9540 & 0.9571 & 0.9527 & 0.9557 & 0.9591 & 0.9561 & 0.9533 \\
\hline scene 5, Case 4 & 0.9310 & 0.9357 & 0.9307 & 0.9351 & 0.9384 & 0.9359 & 0.9340 \\
\hline scene 5 , Case 5 & 0.9073 & 0.9136 & 0.9074 & 0.9137 & 0.9170 & 0.9151 & 0.9136 \\
\hline scene 5 , Case 6 & 0.7925 & 0.8046 & 0.7833 & 0.8087 & 0.8139 & 0.8140 & 0.8117 \\
\hline scene 5 , Case 7 & 0.6957 & 0.7091 & 0.6758 & 0.7186 & 0.7268 & 0.7281 & 0.7222 \\
\hline mean & 0.9011 & 0.9046 & 0.8899 & 0.9050 & 0.9108 & 0.9104 & 0.9034 \\
\hline
\end{tabular}


Hyperspectral image denoising based on low-rank coefficients and orthonormal dictionary

Table 6

Computational time (seconds) of different algorithms, where scenes 1-5 are from Japser Ridge, Cuprite, Urban, Pavia Centre and Washington DC Mall, respectively. Cases 1-7 correspond to noise levels $0.02,0.04,0.06,0.08,0.1,0.2$ and 0.3 , respectively. The mean performances are in bold.

\begin{tabular}{|c|c|c|c|c|c|c|c|}
\hline Cases & BM4D & LRMR & SSTV & NMoG & FastHyDe & SNLRSF & LRCOD \\
\hline scene 1, Case 1 & 87.15 & 7.57 & 85.12 & 33.62 & 0.08 & 10.00 & 7.27 \\
\hline scene 1 , Case 2 & 87.91 & 7.32 & 81.99 & 33.12 & 0.08 & 10.49 & 6.76 \\
\hline scene 1 , Case 3 & 86.80 & 7.23 & 83.55 & 33.41 & 0.08 & 10.40 & 7.00 \\
\hline scene 1 , Case 4 & 86.21 & 7.18 & 82.11 & 33.04 & 0.08 & 9.97 & 5.63 \\
\hline scene 1 , Case 5 & 85.34 & 7.16 & 83.64 & 34.06 & 0.08 & 9.93 & 2.64 \\
\hline scene 1 , Case 6 & 85.42 & 7.27 & 83.98 & 33.98 & 0.11 & 10.60 & 2.30 \\
\hline scene 1 , Case 7 & 87.42 & 7.43 & 82.36 & 33.31 & 0.09 & 11.64 & 2.07 \\
\hline scene 2 , Case 1 & 399.41 & 47.39 & 359.16 & 150.63 & 0.47 & 51.33 & 39.37 \\
\hline scene 2 , Case 2 & 397.43 & 47.48 & 359.12 & 151.86 & 0.46 & 50.85 & 24.24 \\
\hline scene 2 , Case 3 & 397.18 & 47.62 & 359.17 & 151.63 & 0.45 & 50.22 & 18.72 \\
\hline scene 2 , Case 4 & 395.96 & 47.52 & 359.01 & 150.70 & 0.45 & 50.87 & 15.87 \\
\hline scene 2 , Case 5 & 395.75 & 47.67 & 358.92 & 151.27 & 0.45 & 49.80 & 16.72 \\
\hline scene 2 , Case 6 & 393.48 & 48.05 & 359.65 & 151.44 & 0.52 & 49.40 & 22.45 \\
\hline scene 2 , Case 7 & 392.42 & 47.67 & 359.10 & 151.46 & 0.54 & 49.91 & 21.21 \\
\hline scene 3, Case 1 & 694.36 & 92.46 & 636.16 & 277.87 & 0.85 & 97.70 & 122.85 \\
\hline scene 3, Case 2 & 692.11 & 92.30 & 638.49 & 279.67 & 0.82 & 96.51 & 48.73 \\
\hline scene 3 , Case 3 & 698.79 & 94.03 & 649.05 & 284.76 & 0.84 & 100.71 & 32.96 \\
\hline scene 3, Case 4 & 692.60 & 92.87 & 663.28 & 296.85 & 0.86 & 100.29 & 57.96 \\
\hline scene 3 , Case 5 & 697.95 & 93.83 & 667.66 & 287.18 & 0.89 & 100.17 & 15.91 \\
\hline scene 3 , Case 6 & 691.06 & 94.31 & 656.06 & 286.92 & 0.94 & 98.77 & 44.03 \\
\hline scene 3 , Case 7 & 715.76 & 95.72 & 691.53 & 311.69 & 1.07 & 99.14 & 16.96 \\
\hline scene 4, Case 1 & 319.70 & 42.93 & 276.61 & 162.53 & 0.66 & 60.78 & 18.71 \\
\hline scene 4, Case 2 & 318.59 & 42.88 & 276.94 & 160.07 & 0.59 & 59.24 & 25.68 \\
\hline scene 4, Case 3 & 317.88 & 42.93 & 276.77 & 158.37 & 0.56 & 59.37 & 6.84 \\
\hline scene 4 , Case 4 & 317.40 & 42.87 & 276.74 & 162.53 & 0.58 & 59.47 & 7.19 \\
\hline scene 4 , Case 5 & 316.53 & 42.87 & 276.94 & 161.91 & 0.66 & 59.96 & 6.24 \\
\hline scene 4 , Case 6 & 315.40 & 42.83 & 276.86 & 158.27 & 0.66 & 59.28 & 10.10 \\
\hline scene 4, Case 7 & 314.34 & 42.78 & 277.23 & 157.86 & 0.66 & 58.34 & 10.56 \\
\hline scene 5 , Case 1 & 600.23 & 66.37 & 513.66 & 218.15 & 0.64 & 70.18 & 82.38 \\
\hline scene 5 , Case 2 & 600.94 & 66.54 & 514.38 & 218.54 & 0.62 & 70.54 & 17.81 \\
\hline scene 5 , Case 3 & 597.57 & 66.02 & 515.71 & 220.22 & 0.61 & 70.54 & 17.75 \\
\hline scene 5 , Case 4 & 597.19 & 66.46 & 513.62 & 218.80 & 0.61 & 70.55 & 15.42 \\
\hline scene 5 , Case 5 & 596.62 & 66.63 & 514.98 & 218.80 & 0.61 & 71.50 & 18.85 \\
\hline scene 5 , Case 6 & 594.48 & 66.21 & 514.49 & 218.68 & 0.76 & 70.00 & 29.23 \\
\hline scene 5 , Case 7 & 592.20 & 66.39 & 514.81 & 220.65 & 0.76 & 69.58 & 20.31 \\
\hline mean & 418.85 & 51.56 & 378.25 & 170.68 & 0.55 & 57.94 & 23.39 \\
\hline
\end{tabular}


Hyperspectral image denoising based on low-rank coefficients and orthonormal dictionary

Table 7

The rel.ENLs of different algorithms, where scenes 1-5 are from Japser Ridge, Cuprite, Urban, Pavia Centre and Washington DC Mall, respectively. Cases 1-7 correspond to noise levels $0.02,0.04,0.06,0.08,0.1,0.2$ and 0.3 , respectively. The mean performances are in bold.

\begin{tabular}{|c|c|c|c|c|c|c|c|}
\hline Cases & BM4D & LRMR & SSTV & NMoG & FastHyDe & SNLRSF & LRCOD \\
\hline scene 1 , case 1 & 0.0690 & 0.0497 & 0.0798 & 0.0672 & 0.1060 & 0.0069 & 0.0601 \\
\hline scene 1 , case 2 & 0.2352 & 0.0945 & 0.1267 & 0.1409 & 0.2032 & 0.0920 & 0.1506 \\
\hline scene 1 , case 3 & 0.2799 & 0.1726 & 0.1352 & 0.1803 & 0.3030 & 0.1567 & 0.1657 \\
\hline scene 1 , case 4 & 0.2915 & 0.2912 & 0.1554 & 0.1422 & 0.3956 & 0.2107 & 0.2112 \\
\hline scene 1 , case 5 & 0.3152 & 0.2751 & 0.1826 & 0.1618 & 0.4780 & 0.2887 & 0.1780 \\
\hline scene 1 , case 6 & 0.7631 & 0.2963 & 0.3357 & 0.3591 & 1.0192 & 0.7263 & 0.0757 \\
\hline scene 1 , case 7 & 1.3358 & 0.2977 & 0.4824 & 0.5266 & 1.9286 & 1.3676 & 0.0810 \\
\hline scene 2 , case 1 & 0.0383 & 0.0382 & 0.0270 & 0.0676 & 0.1065 & 0.0463 & 0.0319 \\
\hline scene 2 , case 2 & 0.2259 & 0.0133 & 0.0735 & 0.0139 & 0.1645 & 0.1191 & 0.0732 \\
\hline scene 2 , case 3 & 0.2703 & 0.0001 & 0.0425 & 0.0168 & 0.1513 & 0.1315 & 0.0402 \\
\hline scene 2 , case 4 & 0.3454 & 0.0820 & 0.0689 & 0.0331 & 0.1941 & 0.1980 & 0.0197 \\
\hline scene 2 , case 5 & 0.3138 & 0.0003 & 0.0621 & 0.0577 & 0.2028 & 0.2363 & 0.0727 \\
\hline scene 2 , case 6 & 0.5525 & 0.0067 & 0.0351 & 0.0391 & 0.4421 & 0.3938 & 0.4325 \\
\hline scene 2 , case 7 & 0.9284 & 0.1426 & 0.0563 & 0.0875 & 0.7228 & 0.7250 & 0.5737 \\
\hline scene 3 , case 1 & 0.0761 & 0.0441 & 0.0698 & 0.0755 & 0.0611 & 0.0772 & 0.1176 \\
\hline scene 3 , case 2 & 0.1765 & 0.1082 & 0.1186 & 0.1359 & 0.1139 & 0.1464 & 0.1745 \\
\hline scene 3 , case 3 & 0.2883 & 0.1412 & 0.1753 & 0.1863 & 0.1972 & 0.2385 & 0.2068 \\
\hline scene 3 , case 4 & 0.4457 & 0.2451 & 0.2337 & 0.2655 & 0.2924 & 0.3441 & 0.2674 \\
\hline scene 3 , case 5 & 0.5122 & 0.2552 & 0.2779 & 0.3079 & 0.3859 & 0.4420 & 0.2500 \\
\hline scene 3 , case 6 & 1.1741 & 0.7030 & 0.4700 & 0.5186 & 1.0786 & 1.0878 & 0.3007 \\
\hline scene 3 , case 7 & 1.8815 & 0.9022 & 0.5373 & 0.5888 & 1.8733 & 1.7267 & 0.1851 \\
\hline scene 4 , case 1 & 0.1076 & 0.1199 & 0.0550 & 0.0496 & 0.1009 & 0.0643 & 0.1642 \\
\hline scene 4 , case 2 & 0.2461 & 0.2473 & 0.0185 & 0.0427 & 0.2224 & 0.0256 & 0.2771 \\
\hline scene 4 , case 3 & 0.3596 & 0.3120 & 0.0956 & 0.1351 & 0.3247 & 0.1145 & 0.3364 \\
\hline scene 4 , case 4 & 0.5574 & 0.4968 & 0.1833 & 0.2259 & 0.4573 & 0.2153 & 0.4738 \\
\hline scene 4 , case 5 & 0.6376 & 0.5744 & 0.2560 & 0.3071 & 0.5464 & 0.3078 & 0.5149 \\
\hline scene 4 , case 6 & 1.5944 & 1.0767 & 0.7569 & 0.8317 & 1.3370 & 0.9400 & 0.9316 \\
\hline scene 4 , case 7 & 2.4447 & 1.4715 & 1.2286 & 1.3259 & 2.2899 & 1.6218 & 1.2404 \\
\hline scene 5 , case 1 & 0.0376 & 0.0612 & 0.0748 & 0.1153 & 0.0374 & 0.0789 & 0.0094 \\
\hline scene 5 , case 2 & 0.1632 & 0.1220 & 0.1027 & 0.1840 & 0.0630 & 0.1254 & 0.0531 \\
\hline scene 5 , case 3 & 0.2063 & 0.1576 & 0.1433 & 0.2205 & 0.1179 & 0.1856 & 0.0580 \\
\hline scene 5 , case 4 & 0.3064 & 0.1632 & 0.1724 & 0.2574 & 0.1554 & 0.2470 & 0.0234 \\
\hline scene 5 , case 5 & 0.5691 & 0.2791 & 0.1977 & 0.2826 & 0.1816 & 0.3064 & 0.1294 \\
\hline scene 5 , case 6 & 0.9747 & 0.3184 & 0.2700 & 0.3529 & 0.4267 & 0.6100 & 0.1117 \\
\hline scene 5 , case 7 & 1.0567 & 0.2712 & 0.2615 & 0.3107 & 0.6333 & 0.9694 & 0.0731 \\
\hline mean & 0.5652 & 0.2809 & 0.2161 & 0.2461 & 0.4947 & 0.4164 & 0.2304 \\
\hline
\end{tabular}

